\title{
COUNTABLE PARACOMPACTNESS OF PIXLEY-ROY HYPERSPACES
}

\author{
HIDENORI TANAKA \\ (Communicated by Dennis Burke) \\ Dedicated to Professor Yukihiro Kodama on his 60th birthday
}

\begin{abstract}
In this paper, for the Pixley-Roy hyperspace $\mathscr{F}[M]$ of a separable metric space $M$, it will be shown that $\mathscr{F}[M]$ is countably paracompact if and only if $\mathscr{F}[M]^{n}$ is countably paracompact for each $n \in N$ if and only if $M$ is a strong $\Delta$-set. This gives an affirmative answer to D. J. Lutzer's problem.
\end{abstract}

\section{INTRODUCTION}

Throughout this paper, $M$ denotes a metric space. Pixley-Roy hyperspace of the real line was defined by C. Pixley and P. Roy in [3], and later generalized by E. K. van Douwen in [1]. The Pixley-Roy hyperspace $\mathscr{F}[M]$ of a metric space $M$ has as its underlying set the collection of nonempty finite subsets of $M$. If $F \in \mathscr{F}[M]$, then the basic open neighborhoods of $F$ are of the form $[F, U]=\{G \in \mathscr{F}[M]: F \subset G \subset U\}$, where $U$ is an open neighborhood of $F$ in $M$. Then every $\mathscr{F}[M]$ is a zero-dimensional hereditarily metacompact Moore space (see [1]).

$M$ is said to be a $Q$-set if $M$ is separable and every subset of $M$ is an $F_{\sigma}$-set in $M$, and a strong $Q$-set if for each $n \in N, M^{n}$ is a $Q$-set. It is well known that the existence of an uncountable $Q$-set is undecidable in $Z F C$ and is equivalent to the existence of a separable normal nonmetrizable Moore space. T. Przymusinski [5] proved that the existence of an uncountable $Q$-set is equivalent to the existence of an uncountable strong $Q$-set.

Studying countable paracompactness in separable Moore spaces led to the notion of $\Delta$-sets. $M$ is said to be a $\Delta$-set if $M$ is separable and for every decreasing sequence $\left\{A_{n}: n \in N\right\}$ of subsets of $M$ with $\bigcap\left\{A_{n}: n \in N\right\}=\varnothing$, there is a sequence $\left\{U_{n}: n \in N\right\}$ of open subsets of $M$ such that $A_{n} \subset U_{n}$ for each $n \in N$ and $\bigcap\left\{U_{n}: n \in N\right\}=\varnothing$, and a strong $\Delta$-set if for each $n \in N$,

Received by the editors November 14, 1988 and, in revised form, May 24, 1989.

1980 Mathematics Subject Classification (1985 Revision). Primary 54B20, 54D18; Secondary 54E30, 54E35.

Key words and phrases. Pixley-Roy hyperspace, countably paracompact, $\Delta$-set, strong $\Delta$-set, $\delta$-set, almost strong $\delta$-set, strong $\delta$-set. 
$M^{n}$ is a $\Delta$-set. It is clear that every $Q$-set (strong $Q$-set) is a $\Delta$-set (strong $\Delta$-set ). E. K. van Douwen, T. Przymusinski, and G. M. Reed showed that the existence of a separable countably paracompact nonmetrizable Moore space is equivalent to the existence of an uncountable $\Delta$-set (see T. Przymusinski [4]).

Some of the most elegant work involving Pixley-Roy hyperspaces has dealt with problems of normality and countable paracompactness. T. Przymusinski and F. D. Tall [7] proved that if $M$ is a strong $Q$-set, then $\mathscr{F}[M]$ is normal. M. E. Rudin [8] gave a new proof of this result and proved the converse as well: If $M$ is separable, then $\mathscr{F}[M]$ is normal if and only if $M$ is a strong $Q$-set (cf. T. Przymusinski [6]). D. J. Lutzer [2] also proved that if $M$ is a strong $\Delta$-set, then $\mathscr{F}[M]^{n}$ is countably paracompact for each $n \in N$ and stated that it is desirable to prove the converse.

Our purpose of this paper is to give an affirmative answer to this problem. To do so, we introduce some notions of metric spaces and discuss countable paracompactness of Pixley-Roy hyperspaces of metric spaces.

For Pixley-Roy hyperspaces, the reader is referred to E. K. van Douwen [1].

\section{SPECial METRic SPACES}

$M$ is said to be a $q$-set if every subset of $M$ is an $F_{\sigma}$-set in $M$, and a strong $q$-set if for each $n \in N, M^{n}$ is a $q$-set. $M$ is said to be a $\delta$-set if for every decreasing sequence $\left\{A_{n}: n \in N\right\}$ of subsets of $M$ with $\bigcap\left\{A_{n}: n \in N\right\}=\varnothing$, there is a sequence $\left\{U_{n}: n \in N\right\}$ of open subsets of $M$ such that $A_{n} \subset U_{n}$ for each $n \in N$ and $\bigcap\left\{U_{n}: n \in N\right\}=\varnothing$, and a strong $\delta$-set if for each $n \in N, M^{n}$ is a $\delta$-set. Thus every $Q$-set (strong $Q$-set, $\Delta$-set, strong $\Delta$-set) is a separable $q$-set (strong $q$-set, $\delta$-set, strong $\delta$-set).

In order to characterize normality of Pixley-Roy hyperspaces of metric spaces, the author [9] introduced the notion of an almost strong $q$-set.

Let $n \in N$, and let $\tau$ be a permutation of $\{1, \ldots, n\}$. For a point $x=$ $\left(x_{1}, \ldots, x_{n}\right) \in M^{n}$, let $\tau(x)=\left(x_{\tau(1)}, \ldots, x_{\tau(n)}\right)$. A subset $A$ of $M^{n}, n \in N$, is said to be symmetric if for any permutation $\tau$ of $\{1, \ldots, n\}, \tau(A)=A$. A metric space $M$ is said to be an almost strong q-set if for each $n \in N$, every symmetric subset of $M^{n}$ is an $F_{\sigma}$-set in $M^{n}$. The author [9] proved that $\mathscr{F}[M]$ is normal if and only if $\mathscr{F}[M]$ is hereditarily countably paracompact if and only if $M$ is an almost strong $q$-set.

We introduce the notion of an almost strong $\delta$-set. $M$ is said to be an almost strong $\delta$-set if for each $n \in N$, and every decreasing sequence $\left\{A_{m}: m \in N\right\}$ of symmetric subsets of $M^{n}$ with $\bigcap\left\{A_{m}: m \in N\right\}=\varnothing$, there is a sequence $\left\{U_{m}: m \in N\right\}$ of open subsets of $M^{n}$ such that $A_{m} \subset U_{m}$ for each $m \in N$ and $\bigcap\left\{U_{m}: m \in N\right\}=\varnothing$. Clearly every strong $q$-set (strong $\delta$-set) is an almost strong $q$-set (almost strong $\delta$-set) and every almost strong $q$-set (almost strong $\delta$-set $)$ is a $q$-set $(\delta$-set $)$. Furthermore it is obvious that every strong $q$-set (almost strong $q$-set, $q$-set) is a strong $\delta$-set (almost strong $\delta$-set, $\delta$-set). 
The author [9] showed that if $M$ is strongly zero-dimensional (separable), then $M$ is a strong $q$-set (strong $Q$-set) if and only if $M$ is an almost strong $q$-set. The following theorem shows that strong $\delta$-sets and almost strong $\delta$-sets are the same things.

Theorem 2.1. $M$ is a strong $\delta$-set if and only if $M$ is an almost strong $\delta$-set. Proof. It suffices to prove the 'if' part. Assume that $M$ is an almost strong $\delta$-set. Let $n \in N$ and assume that $\left\{A_{m}: m \in N\right\}$ is a decreasing sequence of subsets of $M^{n}$ with $\bigcap\left\{A_{m}: m \in N\right\}=\varnothing$. For each $m \in N$, let $B_{m}=\bigcup\left\{\tau\left(A_{m}\right): \tau\right.$ is a permutation of $\{1, \ldots, n\}\}$. Then $\left\{B_{m}: m \in N\right\}$ is a decreasing sequence of symmetric subsets of $M^{n}$ with $\bigcap\left\{B_{m}: m \in N\right\}=\varnothing$. Since $M$ is an almost strong $\delta$-set, there is sequence $\left\{U_{m}: m \in N\right\}$ of open subsets of $M^{n}$ such that $A_{m} \subset B_{m} \subset U_{m}$ for each $m \in N$ and $\bigcap\left\{U_{m}: m \in N\right\}=\varnothing$. Thus $M$ is a strong $\delta$-set.

Theorem 2.2. Let $f: M \rightarrow M^{\prime}$ be a perfect mapping from a metric space $M$ onto a metric space $M^{\prime}$. If $M$ is a strong $\delta$-set, then $M^{\prime}$ is also a strong $\delta$-set. Proof. For each $n \in N$, let $f^{n}: M^{n} \rightarrow M^{\prime n}$ be a perfect mapping from $M^{n}$ onto $M^{\prime n}$ induced by $f$. Let $n \in N$ and let $\left\{A_{m}: m \in N\right\}$ be a decreasing sequence of subsets of $M^{\prime n}$ with $\bigcap\left\{A_{m}: m \in N\right\}=\varnothing$. For each $m \in N$, let $B_{m}=\left(f^{n}\right)^{-1}\left(A_{m}\right)$. Then $\left\{B_{m}: m \in N\right\}$ is a decreasing sequence of subsets of $M^{n}$ with $\bigcap\left\{B_{m}: m \in N\right\}=\varnothing$. Since $M$ is a strong $\delta$-set, there is a sequence $\left\{V_{m}: m \in N\right\}$ of open subsets of $M^{n}$ such that $B_{m} \subset V_{m}$ for each $m \in N$, and $\bigcap\left\{V_{m}: m \in N\right\}=\varnothing$. Since $f^{n}$ is a perfect mapping, for each $m \in N$, take an open subset $U_{m}$ of $M^{\prime n}$ such that $B_{m} \subset\left(f^{n}\right)^{-1}\left(U_{m}\right) \subset V_{m}$. Then $\left\{U_{m}: m \in N\right\}$ is a sequence of open subsets of $M^{\prime n}$ such that $A_{m} \subset U_{m}$ for each $m \in N$ and $\bigcap\left\{U_{m}: m \in N\right\}=\varnothing$. Thus $M^{\prime}$ is a strong $\delta$-set.

\section{COUNTABle PARACOMPACTNESS}

Let $|A|$ denote the cardinality of a set $A$. For each $n \in N$, let $\mathscr{F}_{n}[M]=$ $\{F \in \mathscr{F}[M]:|F| \leq n\}$. Notice that every $\mathscr{F}_{n}[M]$ is a closed subspace of $\mathscr{F}[M]$ and in particular, $\mathscr{F}[M]$ is a discrete closed subspace of $\mathscr{F}[M]$. Let $d$ be a compatible metric on $M$. For each $F \in \mathscr{F}[M]$, let $B(F, 1 / n)=$ $\bigcup\{B(x, 1 / n): x \in F\}$, where $B(x, 1 / n)=\{y \in M: d(x, y)<1 / n\}$. For each $x=\left(x_{1}, \ldots, x_{n}\right) \in M^{n}, n \in N$, let $F_{x}=\left\{x_{1}, \ldots, x_{n}\right\}$.

We give the main theorem in this paper.

Theorem 3.1. The following are equivalent.

(a) $\mathscr{F}[M]$ is countably paracompact,

(b) $\mathscr{F}[M]^{n}$ is countably paracompact for each $n \in N$,

(c) $M$ is a strong $\delta$-set.

Proof. The implication (b) $\rightarrow$ (a) is obvious and the implication (c) $\rightarrow$ (b) was pointed out by D. J. Lutzer [2, Theorem 4.6] (see also T. Przymusinski and F. D. Tall [7] and the author [9]). 
(a) $\rightarrow$ (c). Assume that $\mathscr{F}[M]$ is countably paracompact. First we shall show that $M$ is a $\delta$-set. Let $d$ be a compatible metric on $M$ bounded by 1. Let $\left\{A_{n}: n \in N\right\}$ be a decreasing sequence of subsets of $M$ with $\bigcap\left\{A_{n}: n \in N\right\}=\varnothing$. For each $n \in N$, let $\mathscr{A}_{n}=\left\{\{x\}: x \in A_{n}\right\}$. Then $\left\{\mathscr{A}_{n}: n \in\right.$ $N\}$ is a decreasing sequence of closed subsets of $\mathscr{F}[M]$ with $\bigcap\left\{\mathscr{A}_{n}: n \in\right.$ $N\}=\varnothing$. Since $\mathscr{F}[M]$ is countably paracompact, there is a decreasing sequence $\left\{\mathscr{U}_{n}: n \in N\right\}$ of open subsets of $\mathscr{F}[M]$ such that $\mathscr{A}_{n} \subset \mathscr{U}_{n}$ for each $n \in N$ and $\bigcap\left\{\mathrm{cl}_{\mathscr{F}_{[M]}} \mathscr{U}_{n}: n \in N\right\}=\varnothing$. For each $n \in N$, let $B_{n}=\{x \in$ $\left.M:[\{x\}, B(x, 1 / n)] \cap \mathscr{U}_{n}=\varnothing\right\}$. Then it is obvious that $M=\bigcup\left\{B_{n}: n \in N\right\}$. For each $n \in N$, we have $\mathrm{cl}_{M} B_{n} \cap A_{n}=\varnothing$. For, assume that $\mathrm{cl}_{M} B_{n} \cap A_{n} \neq \varnothing$ for some $n \in N$. Take a point $x \in \mathrm{cl}_{M} B_{n} \cap A_{n}$. Since $x \in A_{n},\{x\} \in$ $\mathscr{A}_{n} \subset \mathscr{U}_{n}$. Hence there is a $k(\geq n) \in N$ such that $[\{x\}, B(x, 1 / k)] \subset \mathscr{U}_{n}$. Since $x \in \mathrm{cl}_{M} B_{n}, B(x, 1 / k) \cap B_{n} \neq \varnothing$. Take a $y \in B(x, 1 / k) \cap B_{n}$. Then $\{x, y\} \in[\{y\}, B(y, 1 / k)] \cap[\{x\}, B(x, 1 / k)] \subset[\{y\}, B(y, 1 / n)] \cap \mathscr{U}_{n}$, which is a contradiction. Thus for each $n \in N, \mathrm{cl}_{M} B_{n} \cap A_{n}=\varnothing$. It follows that $M$ is a $\delta$-set.

Take an $n \in N$ and assume that for each $i \leq n, M^{i}$ is a $\delta$-set. Let

$$
Z=\left\{\left(x_{1}, \ldots, x_{n+1}\right) \in M^{n+1}: x_{i} \neq x_{j} \text { for each } i, j \leq n+1 \text { and } i \neq j\right\} \text {. }
$$

Then $M^{n+1}$ is a finite union of $Z$ and $G_{\delta}$-sets which are homeomorphic to some $M^{k}(k \leq n)$. In order to prove that $M$ is a strong $\delta$-set, it suffices to prove that $Z$ is a $\delta$-set. By the definition of $Z$, for each $x \in Z,\left|F_{x}\right| \geq 2$. For each $x \in Z$, let $\rho(x)=\left\{d(a, b): a, b \in F_{x}\right.$ and $\left.a \neq b\right\}$. For each $k \in N$, let $Z_{k}=\{x \in Z: 1 /(k+1)<\rho(x) \leq 1 / k\}$. Then $Z_{k} \cap Z_{k^{\prime}}=$ $\varnothing$ for each $k, k^{\prime} \in N$ and $k \neq k^{\prime}$. Let $\left\{A_{m}: m \in N\right\}$ be a decreasing sequence of subsets of $Z$ with $\bigcap\left\{A_{m}: m \in N\right\}=\varnothing$. We may assume $A_{1}=Z$. Furthermore, by the proof of Theorem 2.1, we may assume that every $A_{m}$ is a symmetric subset of $Z$. For each $m, k \in N$, let $A_{m, k}=A_{m} \cap Z_{k}$ and let $\mathscr{A}_{m, k}=\left\{F_{x}: x \in A_{m, k}\right\}$. For each $k \in N,\left\{\mathscr{A}_{m, k}: m \in N\right\}$ is a decreasing sequence of subsets of $\mathscr{F}_{n+1}[M]-\mathscr{F}_{n}[M]$ with $\bigcap\left\{\mathscr{A}_{m, k}: m \in N\right\}=\varnothing$. We show that every $\mathscr{A}_{m, k}$ is a closed subset of $\mathscr{F}[M]$. Fix $m, k \in N$. Since $\mathscr{F}_{n+1}[M]$ is a closed subset of $\mathscr{F}[M], \operatorname{cl}_{\mathscr{F}[M]} \mathscr{A}_{m, k} \subset \mathscr{F}_{n+1}[M]$. Assume that $\mathrm{cl}_{\mathscr{F}[M]} \mathscr{A}_{m, k} \cap \mathscr{F}_{n}[M] \neq \varnothing$. Take an $F=\left\{x_{1}, \ldots, x_{r}\right\} \in \mathrm{cl}_{\mathscr{F}[M]} \mathscr{A}_{m, k} \cap \mathscr{F}_{n}[M]$. Then $r \leq n$. We have $[F, B(F, 1 / 2(k+1))] \cap \mathscr{A}_{m, k} \neq \varnothing$. Take a $y \in A_{m, k}$ such that $F_{y} \in[F, B(F, 1 / 2(k+1))]$. Since $|F|=r \leq n<\left|F_{y}\right|=n+1$, there are $y_{1}, y_{2} \in F_{y}, y_{1} \neq y_{2}$, and an $x \in F$ such that $\left\{y_{1}, y_{2}\right\} \subset B(x, 1 / 2(k+1))$. Thus $d\left(y_{1}, y_{2}\right) \leq d\left(y_{1}, x\right)+d\left(x, y_{2}\right)<1 /(k+1)$, which is a contradiction. Hence $\operatorname{cl}_{\mathscr{F}[M]} \mathscr{A}_{m, k} \cap \mathscr{F}_{n}[M]=\varnothing$. By the definition of topology, $\mathscr{A}_{m, k}$ is a closed subset of $\mathscr{F}[M]$. Since $\mathscr{F}[M]$ is countably paracompact, for each $m$, $k \in N$, there is an open subset $\mathscr{U}_{m, k}$ in $\mathscr{F}[M]$ such that $\mathscr{A}_{m, k} \subset \mathscr{U}_{m, k}$ for each $m, k \in N$ and $\bigcap\left\{\operatorname{cl}_{\mathscr{F}[M]} \mathscr{U}_{m, k}: m \in N\right\}=\varnothing$ for each $k \in N$. We may assume that for each $k \in N,\left\{\mathscr{U}_{m, k}: m \in N\right\}$ is decreasing in $\mathscr{F}[M]$. For each $x \in Z$, there is a unique $k(x) \in N$ such that $x \in Z_{k(x)}$. Let $k \in N$ and 
$x=\left(x_{1}, \ldots, x_{n+1}\right) \in Z_{k}$, that is, $k(x)=k$. Then $F_{x} \in \mathscr{A}_{1, k} \subset \mathscr{U}_{1, k}$. Define $m(x)=\max \left\{m \in N: F_{x} \in \mathscr{U}_{m, k}\right\}$. Choose a real number $\varepsilon(x)$ with $0<\varepsilon(x)<$ $1 /(m(x)+k)$ such that $\left\{B\left(x_{i}, \varepsilon(x)\right): i=1, \ldots, n+1\right\}$ is pairwise disjoint in $M$ and $\left[F_{x}, B\left(F_{x}, \varepsilon(x)\right)\right] \subset \mathscr{U}_{m(x), k}$. (If $x^{\prime}$ is a point of $Z$ such that $F_{x^{\prime}}=F_{x}$ then we take $\varepsilon\left(x^{\prime}\right)$ such that $\varepsilon\left(x^{\prime}\right)=\varepsilon(x)$.) Let $\mathscr{V}_{x}=\left[F_{x}, B\left(F_{x}, \varepsilon(x) / 2\right)\right]$ and $V_{x}=\prod_{i=1}^{n+1} B\left(x_{i}, \varepsilon(x) / 2\right)$. For each $m, k \in N$, let $\mathscr{V}_{m, k}=\bigcup\left\{\mathscr{V}_{x}: x \in A_{m, k}\right\}$ and $V_{m, k}=\bigcup\left\{V_{x}: x \in A_{m, k}\right\}$. For each $m, k \in N, \mathscr{A}_{m, k} \subset \mathscr{V}_{m, k} \subset \mathscr{U}_{m, k}$ and $\left\{\mathscr{V}_{m, k}: m \in N\right\}$ is decreasing in $\mathscr{F}[M]$ for each $k \in N$. Every $V_{m, k}$ is an open subset of $Z$. Let $V_{m}=\bigcup\left\{V_{m, k}: k \in N\right\}$ for $m \in N$. Since $A_{m, k} \subset V_{m, k}$ for $m, k \in N, A_{m} \subset V_{m}$ for $m \in N$. It suffices to prove that $\bigcap\left\{V_{m}: m \in N\right\}=\varnothing$. Take an arbitrary $y=\left(y_{1}, \ldots, y_{n+1}\right) \in Z$. Then there is a $k=k(y) \in N$ such that $y \in Z_{k}$. We show that for each $s(\geq 2(k+1)) \in N, y \notin V_{1, s}$. For, assume that $y \in V_{1, s}$ for some $s(\geq 2(k+1)) \in N$. Then there is an $x=$ $\left(x_{1}, \ldots, x_{n+1}\right) \in A_{1, s}=Z_{s}$ such that $y \in V_{x}$. There are $i, j \leq n+1$ such that $1 /(s+1)<\rho(x)=d\left(x_{i}, x_{j}\right) \leq 1 / s \leq 1 / 2(k+1)$. Since $\varepsilon(x)<1 / s \leq 1 / 2(k+1)$, we have $d\left(y_{i}, x_{i}\right)<1 / 4(k+1)$ and $d\left(y_{j}, x_{j}\right)<1 / 4(k+1)$. Thus $d\left(y_{i}, y_{j}\right) \leq$ $d\left(y_{i}, x_{i}\right)+d\left(x_{i}, x_{j}\right)+d\left(x_{j}, y_{j}\right)<1 / 4(k+1)+1 / 2(k+1)+1 / 4(k+1)=1 /(k+1)$. This is a contradiction. Thus for each $s(\geq 2(k+1)) \in N, y \notin V_{1, s}$. Since $\left\{V_{m, s}: m \in N\right\}$ is decreasing for $s(\geq 2(k+1)) \in N, y \notin V_{m, s}$ for each $m$, $s(\geq 2(k+1)) \in N$. Fix $i<2(k+1)$. Since $\left\{\mathscr{V}_{m, i}: m \in N\right\}$ is a decreasing sequence of open subsets of $\mathscr{F}[M]$ such that $\mathscr{A}_{m, i} \subset \mathscr{V}_{m, i}$ for $m \in N$ and $\bigcap\left\{\operatorname{cl}_{\mathscr{F}[M]} \mathscr{V}_{m, i}: m \in N\right\}=\varnothing$, there is an $s_{i} \in N$ such that $F_{y} \notin \operatorname{cl}_{\mathscr{F}[M]} \mathscr{V}_{s_{i}, i}$. Take an $m_{i} \in N$ with $s_{i} \leq m_{i}$ such that $\left\{B\left(y_{j}, 1 / m_{i}\right): j=1, \ldots, n+1\right\}$ is pairwise disjoint in $M$ and $\left[F_{y}, B\left(F_{y}, 1 / m_{i}\right)\right] \cap \mathscr{V}_{s_{i}, i}=\varnothing$. Assume that $y \in$ $V_{m_{i}, i}$. Then there is an $x \in A_{m_{i}, i}$ such that $y \in V_{x}$. Since $\varepsilon(x)<1 /\left(m_{i}+i\right)$, $F_{y} \cup F_{x} \in\left[F_{y}, B\left(F_{y}, \varepsilon(x) / 2\right)\right] \cap\left[F_{x}, B\left(F_{x}, \varepsilon(x) / 2\right)\right] \subset\left[F_{y}, B\left(F_{y}, 1 / m_{i}\right)\right] \cap \mathscr{V}_{s_{i}, i}$. This is a contradiction. Thus we have $y \notin V_{m_{i}, i}$. Let $m=\max \left\{m_{i}: i<\right.$ $2(k+1)\}$. Then we have $y \notin V_{m}$ and hence, $\bigcap\left\{V_{m}: m \in N\right\}=\varnothing$. It follows that $M$ is a strong $\delta$-set. The proof is completed.

We get an affirmative answer to D. J. Lutzer's problem quoted in the introduction.

Theorem 3.2. If $M$ is separable, then the following are equivalent.

(a) $\mathscr{F}[M]$ is countably paracompact,

(b) $\mathscr{F}[M]^{n}$ is countably paracompact for each $n \in N$,

(c) $M$ is a strong $\Delta$-set.

Proof. This follows from Theorem 3.1 immediately.

Theorem 3.3. Let $f: M \rightarrow M^{\prime}$ be a perfect mapping from a metric space $M$ onto a metric space $M^{\prime}$. If $\mathscr{F}[M]$ is countably paracompact, then $\mathscr{F}\left[M^{\prime}\right]$ is also countably paracompact.

Proof. This immediately follows from Theorems 2.2 and 3.1. 
It does not appear to be known whether, if every finite product of a space $X$ is perfect and countably paracompact, it follows that $X^{\omega}$ is countably paracompact. So we pose the following problem (see D. J. Lutzer [2]).

Problem. If $M$ is a strong $\delta$-set, then is $\mathscr{F}[M]^{\omega}$ countably paracompact?

\section{ACKNOWLEDGMENT}

The author would like to thank the referee for his cheerful instruction and information.

\section{REFERENCES}

1. E. K. van Douwen, The Pixley-Roy topology in spaces of subsets, Set Theoretic Topology, (G. M. Reed, ed.), Academic Press, New York, 1977, pp. 111-134.

2. D. J. Lutzer, Pixley-Roy topology, Topology Proc. 3 (1978), 139-158.

3. C. Pixley and P. Roy, Uncompletable Moore spaces, Proc. Auburn Univ. Conf. (Auburn, Alabama, 1969) (W. R. R. Transue, ed.), 1969, pp. 75-85.

4. T. Przymusinski, Normality and separability of Moore spaces, Set Theoretic Topology (G. M. Reed, ed.), Academic Press, New York, 1977, pp. 325-337.

5. _ The existence of $Q$-sets is equivalent to the existence of strong $Q$-sets, Proc. Amer. Math. Soc. 79 (1980), 626-628.

6. __ Normality and paracompactness of Pixley-Roy hyperspaces, Fund. Math. 113 (1981), 201-219.

7. T. Przymusinski and F. D. Tall, The undecidability of the existence of a non-separable normal Moore space satisfying the countable chain condition, Fund. Math. 85 (1974), 291-297.

8. M. E. Rudin, Pixley-Roy and the Souslin line, Proc. Amer. Math. Soc. 74 (1979), 128-134.

9. H. Tanaka, Normality and hereditary countable paracompactness of Pixley-Roy hyperspaces, Fund. Math. 126 (1986), 201-208.

Department of Management and Information Science, Jobu University, Shimmachi, TANO-GUN, GUNMA, 370-13, JAPAN 